\title{
Isotope effects on delayed annihilation time spectra of antiprotonic helium atoms in a low-temperature gas
}

\author{
B. Ketzer, F. J. Hartmann, H. Daniel, T. von Egidy, A. Niestroj, S. Schmid, and W. Schmid
}

Physik Department, Technische Universität München, D-85747 Garching, Germany

T. Yamazaki, I. Sugai, and K. Nakayoshi

Institute for Nuclear Study, University of Tokyo, 3-2-1 Midori-cho, Tanashi, Tokyo 188, Japan

R. S. Hayano, F. E. Maas, H. A. Torii, T. Ishikawa, and H. Tamura

Department of Physics, University of Tokyo, 7-3-1 Hongo, Bunkyo-ku, Tokyo 113, Japan

\author{
N. Morita \\ Institute for Molecular Science, Myodaiji, Okazaki 444, Japan
}

D. Horváth

Central Research Institute for Physics, Research Institute for Particle and Nuclear Physics, H-1525 Budapest, Hungary

\author{
J. Eades and E. Widmann \\ CERN, CH-1211 Geneva 23, Switzerland
}

(Received 5 October 1995)

\begin{abstract}
The delayed annihilation time spectra (DATS) of antiprotonic helium atoms have been studied in isotopically pure low-temperature ${ }^{3} \mathrm{He}$ and ${ }^{4} \mathrm{He}$ gas at various densities. The DATS taken at $5.8 \mathrm{~K}$ and 400 mbar are very similar in shape except for (i) a small difference in the time scale and (ii) the presence of a distinct fast decay component in the case of ${ }^{3} \mathrm{He}$. The ratio of overall trapping times (mean lifetimes against annihilation), $R=T_{\text {trap }}\left({ }^{4} \mathrm{He}\right) / T_{\text {trap }}\left({ }^{3} \mathrm{He}\right)$, has been determined to be $1.144 \pm 0.009$, which is in good agreement with a theoretical estimate yielding $R=\left[M^{*}\left(\bar{p}^{4} \mathrm{He}\right) / M^{*}\left(\bar{p}^{3} \mathrm{He}\right)\right]^{2}=1.14$, where $M^{*}$ denotes the reduced mass of the $\bar{p} \mathrm{He}^{2+}$ system. The presence of a short-lived component with a lifetime of $0.154 \pm 0.007 \mu \mathrm{s}$ in the case of ${ }^{3} \mathrm{He}$ suggests that the $\bar{p}{ }^{3} \mathrm{He}^{+}$atom has a state of intermediate lifetime on the border between a metastable zone and an Auger-dominated short-lived zone. The fraction of antiprotons trapped in metastable states at $5.8 \mathrm{~K}$ and 400 mbar is lower by $22.2(4) \%$ for ${ }^{3} \mathrm{He}$ than for ${ }^{4} \mathrm{He}$. All the data can be fitted fairly well with simple three-level and four-level cascade models.
\end{abstract}

PACS number(s): $36.10 .-\mathrm{k}$

\section{INTRODUCTION}

The discovery of the delayed annihilation of antiprotons in liquid helium at the Japanese National Laboratory for High Energy Physics, KEK, in 1991 [1] contradicted the long-standing belief that every antiproton coming to rest in matter would annihilate with the nucleus within picoseconds. About $3 \%$ of all antiprotons stopped in liquid helium were found to survive for an average lifetime of $3 \mu \mathrm{s}$, some of them living as long as $25 \mu \mathrm{s}$ - more than seven orders of magnitude above the expected value. The historical background is reviewed in [2].

The first experiment at the low energy antiproton ring (LEAR) at CERN in November of 1991 confirmed this longevity in gaseous ${ }^{3} \mathrm{He}$ and ${ }^{4} \mathrm{He}$ at room temperature, and revealed also a different mean lifetime of the long-living antiprotons in the two helium isotopes [3,4]. This isotope effect had previously been postulated by Yamazaki and Ohtsuki [5] on the basis of a $\bar{p} \mathrm{He}^{+}$model suggested by Condo [6] and developed by Russell [7]. According to this model a metastable $\bar{p} \mathrm{He}^{+}$atom results when the antiproton is captured into nearly circular states with high principal quantum number

$$
n \sim n_{0} \equiv \sqrt{M^{*} / m_{\mathrm{e}}}
$$

and high angular momentum quantum number $l \leqq n-1$. Depending on the reduced mass $M^{*}$ of the $\bar{p} \mathrm{He}^{2+}$ system, $n_{0}$ is 38 for $\bar{p}{ }^{4} \mathrm{He}^{+}$and 37 for $\bar{p}^{3} \mathrm{He}^{+}$. The small energy spacing of levels with $\Delta n=1$ in this region $(\sim 2 \mathrm{eV})$ strongly suppresses internal and external Auger deexcitation, since an energy of at least $25 \mathrm{eV}$ is necessary for electron emission. The continued electron presence removes the $l$ degeneracy of antiproton states with equal $n$. At the same time the electron provides a Pauli-repulsion effect during collisions with other helium atoms. Both these effects strongly reduce the collisional Stark mixing to $n S$ states, which normally produces immediate annihilation. The only remaining deexcitation processes are slow radiative transitions. The joint effect of all these circumstances is to produce the anomalously long antiproton lifetimes observed.

In November 1992 further extensive measurements of delayed annihilation time spectra (DATS) from lowtemperature ${ }^{4} \mathrm{He}$ gas, high-pressure liquid ${ }^{3} \mathrm{He}$ and ${ }^{4} \mathrm{He}$, and solid ${ }^{4} \mathrm{He}$ were carried out, which yielded important results on the phase and density dependence of DATS from pure helium media [8]. The isotope effect seen previously in 


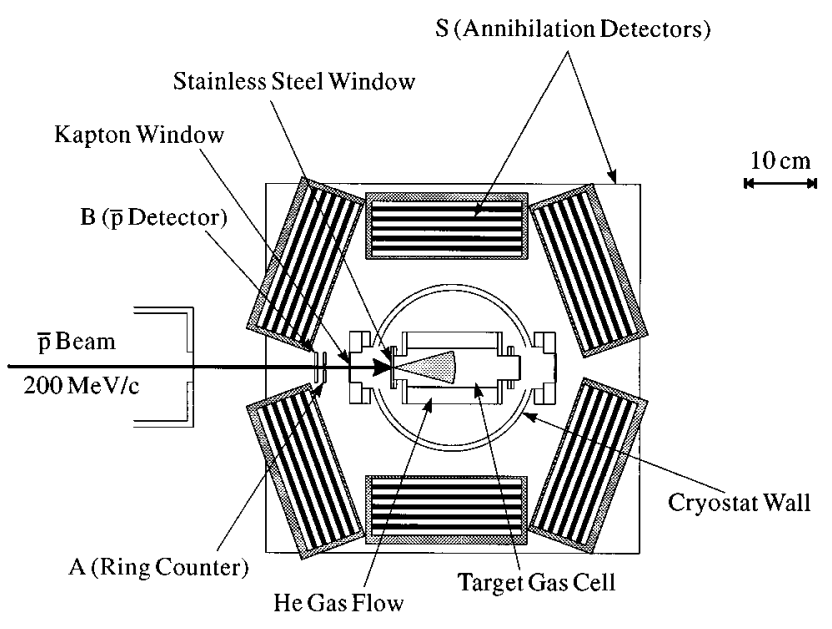

FIG. 1. Top view of the experimental setup. The $\bar{p}$, extracted with a momentum of $p=200 \mathrm{MeV} / c$, traverse a beam telescope ( $B$ and $A$ ) before entering the target chamber through a steel window. A liquid helium cryostat keeps the chamber at temperatures between $4.3 \mathrm{~K}$ and $10 \mathrm{~K}$. Both charged and neutral annihilation products are detected by seven shower counters $S$, six of them surrounding the cryostat and one positioned underneath.

room-temperature gases [4] was found to occur in the liquid phase also. In liquid ${ }^{4} \mathrm{He}$ the lifetime gradually became shorter with increasing density and a distinct fast component was observed. The DATS from low-temperature ${ }^{4} \mathrm{He}$ gas showed the longest $\bar{p}$ lifetime $(\sim 4 \mu \mathrm{s})$ and no fastdecaying component. These observations prompted us to look for a similar isotope effect in low-temperature gas.

In the present paper we report the results of precise measurements of DATS from low-temperature ${ }^{3} \mathrm{He}$ and ${ }^{4} \mathrm{He}$ gases. Here, we employed a new method using a highly efficient detection system for annihilation pions (both charged and neutral), which was designed and developed for laser resonance experiments $[9,10]$.

After a brief account of the experimental setup in Sec. II the experimental results are presented in Sec. III. Section IV introduces some models to reproduce the characteristic shape of the time spectra. In Sec. V the experimental results are discussed and compared with theoretical accounts.

\section{EXPERIMENTAL METHOD}

Here we confine ourselves to a brief description of the setup, the electronics, and the data acquisition system necessary to record the time spectra.

\section{A. Experimental arrangement}

Figure 1 shows a plan view of the setup used in the experiments for investigating the long-lived states in $\bar{p} \mathrm{He}^{+}$atoms. A $200 \mathrm{MeV} / c \bar{p}$ beam with an intensity of about $10^{4} \bar{p} \mathrm{~s}^{-1}$ was delivered from LEAR. At the end of the beam line, the antiprotons passed through a $100 \mu \mathrm{m}$ thick Be window into the air and then traversed a $0.5 \mathrm{~mm}$ thick plastic scintillator $(B)$, which served as a beam counter to provide the start signal for the annihilation time measurements. After passing through the hole in a ring veto counter $(A)$, the antiprotons entered the helium target chamber through $50 \mu \mathrm{m}$ Kapton and $407 \mu \mathrm{m}$ steel windows, the latter being covered with an $875 \mu \mathrm{m}$ thick Euplex foil (similar to Kapton) for moderation, a value which was determined by Monte Carlo simulation. Details on this program, which takes into account the Barkas effect of the energy loss, can be found in [4]. A charge-coupled device (CCD) camera with its optical axis at $45^{\circ}$ to the beam axis imaged scintillation light leaking out of the surface of the beam counter $B$ and allowed us to monitor the beam position [11]. An additional $50 \mu \mathrm{m}$ thick degrader (Euplex foil) was placed in front of the Kapton window to fine tune the stopping distribution of antiprotons in the target gas.

Annihilation time spectra were recorded in isotopically pure gaseous ${ }^{4} \mathrm{He}$ (impurity content $<1 \mathrm{ppm}$ ) and ${ }^{3} \mathrm{He}$ (enrichment $99.95 \%$, impurity content $<30 \mathrm{ppm}$ ) at pressures between 200 mbar and 1040 mbar and low temperatures, since the longest $\bar{p}$ lifetimes have been observed under these conditions [8].

The cryostat used to cool the target gas to temperatures between $4.3 \mathrm{~K}$ and $10 \mathrm{~K}$ resembled the one used in previous experiments discussed in [8]: the cylindrical target chamber was $21.8 \mathrm{~cm}$ long and had a diameter of $6.2 \mathrm{~cm}$. Liquid helium from a reservoir evaporated into a second chamber surrounding the target chamber, the flow of helium being controlled by a needle valve. The fine adjustment of the temperature was carried out via a heating wire located at the vaporizer and connected in a feedback loop to a temperature sensor in contact with the target vessel.

Charged pions and $\pi^{0}$ decay $\gamma$ 's from antiproton annihilation were detected in seven shower counters ( $S$ in Fig. 1), each comprising 11 layers of alternating scintillator and lead plates. These counters, subtending a solid angle of about $80 \%$ of $4 \pi$, were able to detect annihilation events with an efficiency of about $99.7 \%$.

\section{B. Electronics}

To measure the time delay between an incoming antiproton ( $B$ counter hit) and the arrival of the corresponding annihilation products ( $S$ counter hits), a wide range TDC (Time to Digital Converter, LeCroy model 4208) with a resolution of $1 \mathrm{~ns}$ was used. Light from the beam counter $B$ was detected by two photomultipliers on opposite sides of the scintillator. The coincidence of these beam detectors $B 1$ and $B 2$ started all the TDC channels if the antiproton had not hit the veto counter $A$, if the computer was not busy with readout, and if no $\bar{p}$ had been detected by either $A, B 1$, or $B 2$ within the previous $50 \mu \mathrm{s}$. Each shower counter signal stopped a different TDC channel. The trigger condition for computer readout of an event was

$$
B 1 * B 2 * \bar{A} * \overline{\text { Computer veto }} * \overline{\text { Prepileup }} * \sum_{i=0}^{6} S_{i},
$$

where * refers to "and," the overbar refers to "not" and $\sum_{i=0}^{6} S_{i}$ is the analog sum of the seven shower counter signals. The time spectra were recorded in the range from $-0.5 \mu \mathrm{s}$ to $+49.5 \mu \mathrm{s}$ relative to the time of the prompt annihilation peak by delaying the stop signals by $\sim 500 \mathrm{~ns}$. Prompt annihilation events could be discarded by the electronics ("prompt veto"), thus considerably reducing the 
TABLE I. Summary of target conditions, trapping fractions, average lifetimes, and fitted decay rates for low-temperature pure ${ }^{3} \mathrm{He}$ measurements. The errors quoted for temperature and pressure arise from fluctuations in time around the mean value, caused by our feedback stabilizing system.

\begin{tabular}{|c|c|c|c|c|c|c|c|c|}
\hline \multirow{2}{*}{$\begin{array}{l}T \\
(\mathrm{~K}) \\
5.83(20)\end{array}$} & \multirow{2}{*}{$\begin{array}{c}\begin{array}{c}p \\
(\mathrm{mbar})\end{array} \\
204.5(21)\end{array}$} & \multirow{2}{*}{$\frac{\begin{array}{c}\rho_{\mathrm{mol}} \\
\left(10^{-4} \mathrm{~mol} / \mathrm{cm}^{3}\right)\end{array}}{4.28(16)}$} & \multirow{2}{*}{$\begin{array}{c}f \\
(\%) \\
0.320(4)\end{array}$} & \multirow{2}{*}{$\begin{array}{c}T_{\mathrm{av}}(2 \mu \mathrm{s}) \\
(\mu \mathrm{s}) \\
3.48(12)\end{array}$} & \multicolumn{2}{|c|}{$\begin{array}{c}\text { Three-level model } \\
\lambda_{1}\left(\mu \mathrm{s}^{-1}\right) \lambda_{3}\left(\mu \mathrm{s}^{-1}\right)\end{array}$} & \multicolumn{2}{|c|}{$\begin{array}{c}\text { Four-level model } \\
\lambda_{1}\left(\mu \mathrm{s}^{-1}\right) \lambda_{4}\left(\mu \mathrm{s}^{-1}\right)\end{array}$} \\
\hline & & & & & $0.404(8)$ & $1.150(75)$ & $0.556(12)$ & $3.46(45)$ \\
\hline $4.81(18)$ & $193.5(7)$ & $4.95(19)$ & $0.594(5)$ & $3.41(7)$ & $0.411(4)$ & $1.206(46)$ & $0.551(7)$ & $2.76(18)$ \\
\hline $5.86(18)$ & $402.7(56)$ & $8.52(30)$ & & $3.34(2)$ & $0.401(1)$ & $1.414(14)$ & $0.529(2)$ & $2.602(41)$ \\
\hline $5.83(14)$ & $406.3(54)$ & $8.65(25)$ & & $3.36(1)$ & $0.400(1)$ & $1.428(7)$ & $0.521(1)$ & $2.448(19)$ \\
\hline $5.83(17)$ & $409(16)$ & $8.70(43)$ & & $3.35(2)$ & $0.402(1)$ & $1.401(15)$ & $0.527(2)$ & $2.488(43)$ \\
\hline $5.83(13)$ & $409.5(4)$ & $8.72(20)$ & $2.217(19)$ & $3.38(8)$ & $0.406(4)$ & $1.347(48)$ & $0.521(9)$ & $2.13(12)$ \\
\hline $5.83(13)$ & $409.8(4)$ & $8.72(20)$ & $2.235(10)$ & $3.38(4)$ & $0.402(2)$ & $1.451(28)$ & $0.526(4)$ & $2.561(75)$ \\
\hline $5.83(13)$ & $552.1(5)$ & $11.89(28)$ & & $3.37(2)$ & $0.399(1)$ & $1.655(20)$ & $0.507(3)$ & $2.511(43)$ \\
\hline $5.83(14)$ & $552.7(7)$ & $11.90(30)$ & $2.369(10)$ & $3.37(4)$ & $0.403(2)$ & $1.574(31)$ & $0.516(4)$ & $2.482(69)$ \\
\hline $5.83(13)$ & $553.0(20)$ & $11.91(28)$ & & $3.34(2)$ & $0.401(1)$ & $1.624(15)$ & $0.513(2)$ & $2.514(33)$ \\
\hline $5.83(16)$ & $565.5(69)$ & $12.19(38)$ & $2.368(7)$ & $3.37(3)$ & $0.401(1)$ & $1.613(21)$ & $0.508(3)$ & $2.421(44)$ \\
\hline $5.83(10)$ & $566.5(87)$ & $12.22(29)$ & & $3.36(1)$ & $0.399(1)$ & $1.655(13)$ & $0.508(2)$ & $2.535(27)$ \\
\hline
\end{tabular}

computer dead time. To avoid confusion in assigning annihilation events to the corresponding antiprotons, prepileup events (those in which a $\bar{p}$ had been detected within the previous $50 \mu \mathrm{s}$ ) were rejected by hardware veto, as described above. Postpileup events (events with a second $\bar{p}$ within $50 \mu \mathrm{s}$ after TDC start), on the other hand, had to be discarded in the analysis. For that purpose the time spectra of the beam detectors $B 1$ and $B 2$ were recorded using the multihit mode of the TDC (in which the time interval between two successive pulses in the same input line is digitized). If the spectrum of one of the $B$ counters had multiple hits within the time window of $50 \mu \mathrm{s}$ the event was discarded by software.

For each event the TDC information as well as the analog signals of all detectors were stored. The data were acquired by a CES Starburst J11 CAMAC module, a PDP-11 compatible CAMAC Auxiliary Controller, transferred to a VAX computer station using direct memory access and then written on magnetic tape. Since we carried out prepileup and postpileup rejection in an interval of $50 \mu \mathrm{s}$ each, the dead time of the data acquisition system was $\sim 100 \mu$ s per accepted event.

\section{Data analysis}

The data were analyzed event by event as follows.

(1) Postpileup events were rejected using the multihit mode of the TDC.

(2) The annihilation time and the time spread of the shower counter hits were calculated.

(3) Events with only a single hit on one of the seven shower counters were discarded.

The postpileup rejection has already been described in the preceding section. The annihilation time is defined as the time between passage of the $\bar{p}$ through detector $B$ and the average time of arrival of its annihilation products in the shower counters. Only events for which the standard deviation of the shower counter events from their average was less than $10 \mathrm{~ns}$ were included ("dispersion" cut). To minimize the background caused by $\pi^{+} \rightarrow \mu^{+} \rightarrow e^{+}$decays, we required that at least two of the seven shower counters had detected an annihilation product ("multiplicity two" cut).

\section{EXPERIMENTAL RESULTS}

Figure 2 shows two typical DATS from ${ }^{4} \mathrm{He}$ and ${ }^{3} \mathrm{He}$, both taken at $5.8 \mathrm{~K}$ and $400 \mathrm{mbar}$. The time spectrum from ${ }^{4} \mathrm{He}$ is essentially the same as our previous one $[3,4,8]$, but with better statistics and background suppression, showing again that antiprotons can survive up to $25 \mu \mathrm{s}$ in helium gas. Both DATS clearly deviate from a single exponential slope, exhibiting a downward bent structure for long decay times when plotted in a logarithmic scale. This shape indicates that the $\bar{p}$ cascades down a ladder of successive metastable levels, before coming to a state from which a fast Auger transi-

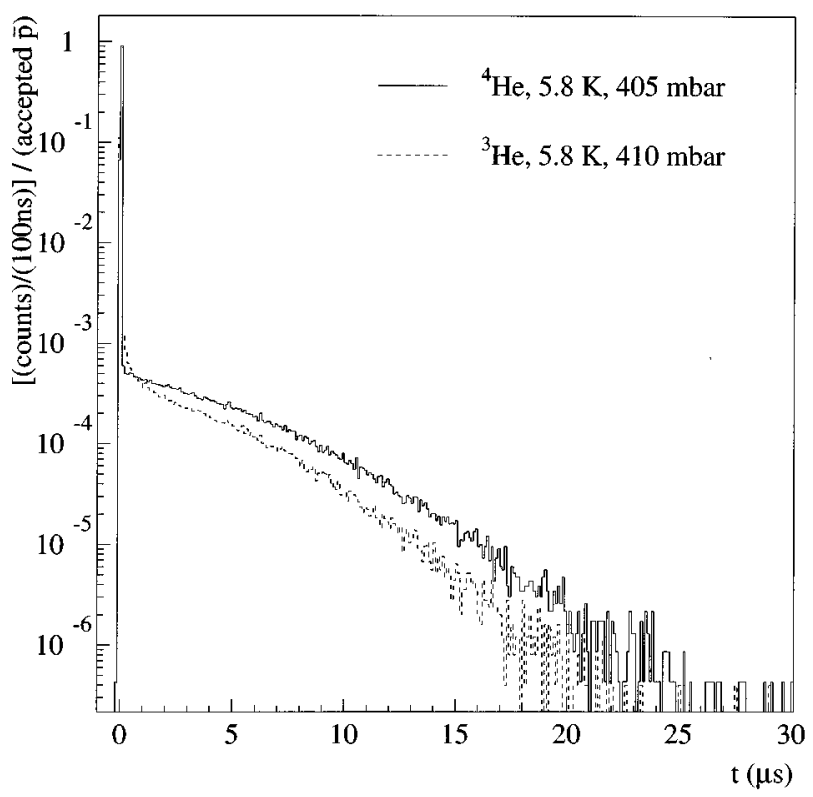

FIG. 2. Two typical $\bar{p}$ delayed annihilation time spectra (DATS) from ${ }^{4} \mathrm{He}$ and ${ }^{3} \mathrm{He}$, showing the downward bent structure in the logarithmic plot, the different overall trapping times of antiprotons, and the fast-decaying component in the case of ${ }^{3} \mathrm{He}$. 
TABLE II. Summary of target conditions, trapping fractions, average lifetimes, and fitted decay rates for low-temperature pure ${ }^{4} \mathrm{He}$ measurements. The errors for $T$ and $p$ are due to fluctuations in time around the mean value.

\begin{tabular}{|c|c|c|c|c|c|c|c|c|}
\hline \multirow{2}{*}{$\begin{array}{l}T \\
(\mathrm{~K}) \\
5.83(10)\end{array}$} & \multirow{2}{*}{$\begin{array}{c}\begin{array}{c}p \\
(\mathrm{mbar})\end{array} \\
204.2(3)\end{array}$} & \multirow{2}{*}{$\frac{\begin{array}{c}\rho_{\mathrm{mol}} \\
\left(10^{-4} \mathrm{~mol} / \mathrm{cm}^{3}\right)\end{array}}{4.30(8)}$} & \multirow{2}{*}{$\begin{array}{c}f \\
(\%) \\
1.094(9)\end{array}$} & \multirow{2}{*}{$\begin{array}{c}T_{\mathrm{av}}(2.3 \mu \mathrm{s}) \\
(\mu \mathrm{s}) \\
4.05(8)\end{array}$} & \multicolumn{2}{|c|}{$\begin{array}{c}\text { Three-level model } \\
\lambda_{1}\left(\mu \mathrm{s}^{-1}\right) \lambda_{3}\left(\mu \mathrm{s}^{-1}\right)\end{array}$} & \multicolumn{2}{|c|}{$\begin{array}{c}\text { Four-level model } \\
\lambda_{1}\left(\mu \mathrm{s}^{-1}\right) \lambda_{4}\left(\mu \mathrm{s}^{-1}\right)\end{array}$} \\
\hline & & & & & $0.383(7)$ & $0.576(15)$ & $0.487(8)$ & $1.006(65)$ \\
\hline $5.82(19)$ & $404.5(19)$ & $8.73(30)$ & $2.866(11)$ & $3.88(4)$ & $0.387(3)$ & $0.587(7)$ & $0.468(5)$ & $0.795(24)$ \\
\hline $5.83(10)$ & $425.1(15)$ & $9.18(17)$ & & $3.88(3)$ & $0.383(2)$ & $0.609(5)$ & $0.452(5)$ & $0.762(17)$ \\
\hline $10.26(12)$ & $805.8(42)$ & $9.64(13)$ & $2.931(9)$ & $3.87(3)$ & $0.381(2)$ & $0.621(5)$ & $0.447(5)$ & $0.759(18)$ \\
\hline $5.02(12)$ & $392.1(5)$ & $9.98(25)$ & $2.942(10)$ & $3.88(3)$ & $0.383(2)$ & $0.616(6)$ & $0.457(5)$ & $0.795(19)$ \\
\hline $5.83(10)$ & $548(17)$ & $12.01(46)$ & & $3.85(2)$ & $0.376(1)$ & $0.668(4)$ & $0.442(3)$ & $0.820(12)$ \\
\hline $10.26(12)$ & $1000.0(8)$ & $12.02(14)$ & $2.943(10)$ & $3.86(3)$ & $0.371(2)$ & $0.706(7)$ & $0.429(6)$ & $0.833(20)$ \\
\hline $10.26(11)$ & $1002.0(10)$ & $12.04(13)$ & $2.937(12)$ & $3.84(4)$ & $0.378(3)$ & $0.684(8)$ & $0.449(6)$ & $0.855(25)$ \\
\hline $5.83(18)$ & $559.7(24)$ & $12.28(41)$ & $2.874(19)$ & $3.78(6)$ & $0.392(4)$ & $0.669(13)$ & $0.469(10)$ & $0.862(40)$ \\
\hline $5.82(18)$ & $560.0(23)$ & $12.31(41)$ & $2.926(19)$ & $3.85(6)$ & $0.388(4)$ & $0.671(13)$ & $0.468(8)$ & $0.888(37)$ \\
\hline $5.82(18)$ & $560.2(23)$ & $12.31(41)$ & $2.932(20)$ & $3.81(6)$ & $0.390(4)$ & $0.692(13)$ & $0.467(9)$ & $0.889(40)$ \\
\hline $5.82(18)$ & $560.2(21)$ & $12.31(41)$ & $2.870(45)$ & $3.78(14)$ & $0.396(11)$ & $0.699(32)$ & $0.461(70)$ & $0.84(16)$ \\
\hline $10.27(11)$ & 1041.0(1) & $12.51(14)$ & $2.873(29)$ & $3.95(10)$ & $0.378(6)$ & $0.759(23)$ & $0.457(14)$ & $0.983(66)$ \\
\hline $10.26(10)$ & $1041.0(1)$ & $12.52(13)$ & $2.922(10)$ & $3.82(3)$ & $0.373(2)$ & $0.755(7)$ & $0.443(5)$ & $0.932(20)$ \\
\hline $5.83(18)$ & $609.8(38)$ & $13.46(46)$ & $2.928(10)$ & $3.86(3)$ & $0.372(2)$ & $0.699(7)$ & $0.443(6)$ & $0.872(21)$ \\
\hline $5.83(10)$ & $644.1(60)$ & $14.27(30)$ & & $3.89(3)$ & $0.367(2)$ & $0.722(8)$ & $0.432(6)$ & $0.874(22)$ \\
\hline $5.02(12)$ & $601.3(11)$ & $15.89(42)$ & $2.911(11)$ & $3.85(4)$ & $0.367(2)$ & $0.775(9)$ & $0.433(6)$ & $0.941(24)$ \\
\hline $5.83(18)$ & $806.2(39)$ & $18.24(63)$ & $2.900(11)$ & $3.85(4)$ & $0.362(2)$ & $0.868(10)$ & $0.432(6)$ & $1.071(25)$ \\
\hline $5.02(12)$ & $826.7(73)$ & $22.89(69)$ & $2.884(16)$ & $3.88(5)$ & $0.354(3)$ & $0.995(18)$ & $0.438(6)$ & $1.328(42)$ \\
\hline $5.82(17)$ & $1009.0(45)$ & $23.54(80)$ & $2.881(14)$ & $3.91(5)$ & $0.349(2)$ & $1.015(15)$ & $0.420(6)$ & $1.273(35)$ \\
\hline $5.82(18)$ & $1041.0(1)$ & $24.40(87)$ & $2.854(12)$ & $3.86(4)$ & $0.350(2)$ & $1.166(17)$ & $0.427(5)$ & $1.503(35)$ \\
\hline $5.02(12)$ & $986.0(49)$ & $28.36(87)$ & $2.838(19)$ & $3.86(6)$ & $0.357(3)$ & $1.070(24)$ & $0.448(7)$ & $1.476(52)$ \\
\hline $5.02(12)$ & $990.4(29)$ & $28.51(86)$ & $2.927(41)$ & $3.84(13)$ & $0.362(7)$ & $1.230(60)$ & $0.435(20)$ & $1.53(12)$ \\
\hline $5.02(13)$ & $992.0(23)$ & $28.57(93)$ & $2.878(11)$ & $3.90(4)$ & $0.348(2)$ & $1.121(15)$ & $0.431(4)$ & $1.498(33)$ \\
\hline
\end{tabular}

tion is possible. Annihilation via the Stark effect in a collision with another helium atom then immediately follows the ejection of the remaining electron. Although the maximum trapping time of an antiproton in ${ }^{4} \mathrm{He}$ is clearly longer than in ${ }^{3} \mathrm{He}$, the DATS from ${ }^{3} \mathrm{He}$ and ${ }^{4} \mathrm{He}$ are very similar in shape for $t>2 \mu \mathrm{s}$. For shorter decay times, however, a distinct fast component shows up in ${ }^{3} \mathrm{He}$, but not in ${ }^{4} \mathrm{He}$.

Tables I and II summarize the experimental conditions and results for low-temperature pure ${ }^{3} \mathrm{He}$ and ${ }^{4} \mathrm{He}$ measurements. The molar density $\rho_{\text {mol }}$ was calculated using the second order virial expansion of the state equation. The second virial coefficients for ${ }^{3} \mathrm{He}$ and ${ }^{4} \mathrm{He}$ were interpolated from theoretical values [12]. The trapping fraction $f$, the average lifetime $T_{\mathrm{av}}$ of antiprotons, and the results of fitting cascade models to the time spectra, shown in the subsequent columns, will be discussed in the following sections.

\section{A. Trapping fraction}

The trapping fraction $f$, defined as

$$
f=\frac{\text { (number of delayed annihilations) }}{\text { (total number of annihilations) }}
$$

is only given for runs in which the prompt peak had not been suppressed by the electronic prompt veto. An annihilation was then considered as delayed if it occurred more than 15 ns after arrival of the antiproton. In Fig. 3 the experimental results for $f$ in ${ }^{3} \mathrm{He}$ and ${ }^{4} \mathrm{He}$, shown in Tables I and II, are plotted versus the molar density. The trapping fraction in ${ }^{3} \mathrm{He}$ is always significantly lower than that in ${ }^{4} \mathrm{He}$. At $5.8 \mathrm{~K}$ and 400 mbar the observed ratio is

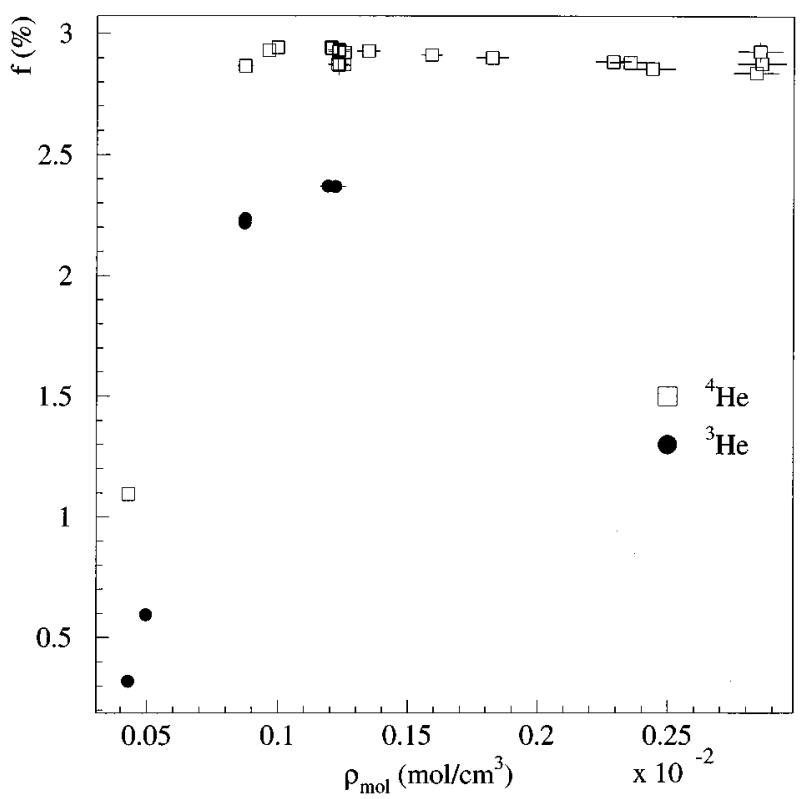

FIG. 3. Trapping fraction of antiprotons in ${ }^{3} \mathrm{He}$ and ${ }^{4} \mathrm{He}$ at low pressures as a function of the molar density. 

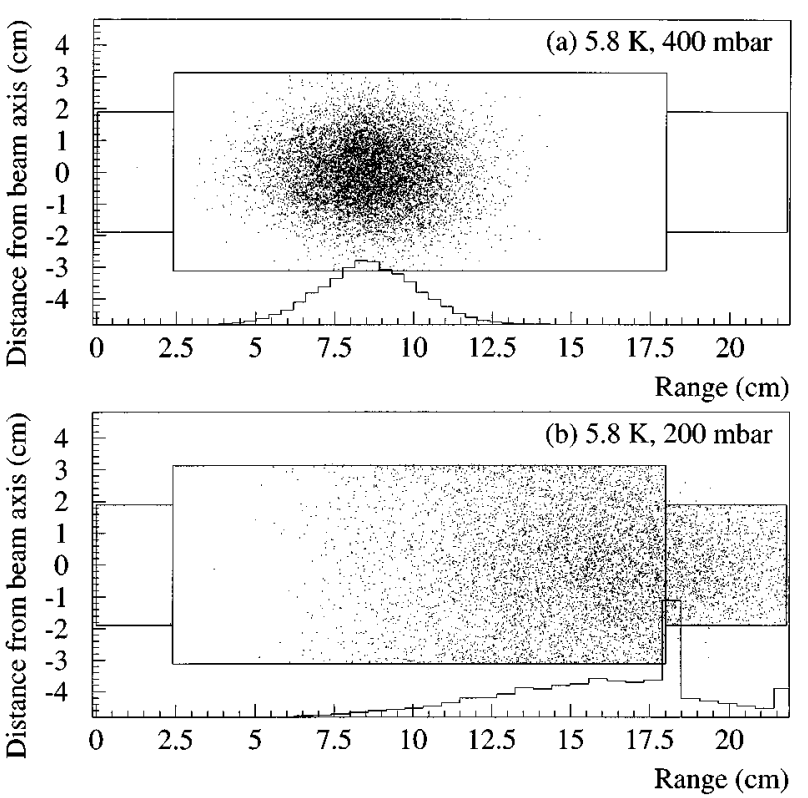

FIG. 4. Scatter plot of the calculated stopping distribution of $10000 \bar{p}$ in the He target at (a) $5.8 \mathrm{~K}, 400$ mbar and (b) $5.8 \mathrm{~K}, 200$ mbar. The antiprotons enter the target chamber (indicated by solid lines in the plot, cf. Fig. 1) from the left side. At 400 mbar all antiprotons are stopped in the target gas, while at 200 mbar a large fraction was found to stop in the walls of the target cell, considerably reducing the fraction of delayed annihilations. The projection of the stopping distribution onto the beam axis (range distribution) is shown as a histogram.

$$
\frac{f\left({ }^{3} \mathrm{He}\right)}{f\left({ }^{4} \mathrm{He}\right)}=0.778 \pm 0.004
$$

With the exception of very low densities it remains constant in the density region under investigation. The apparent decrease of the trapping fraction at low densities is an artifact caused by our experimental setup as will be explained in the following paragraph.

The scintillation counter array used in previous experiments $[4,8]$ was able to distinguish between annihilations in the target gas and in the walls of the target chamber. This feature was lost in the highly efficient shower counter system described in Sec. II. Antiprotons annihilating in the target chamber walls, however, would contribute to the prompt peak and would therefore bias the result for the trapping fraction. To investigate this problem, we calculated the stopping distribution of antiprotons in our target by Monte Carlo simulation [4]. Figure 4(a) shows the stopping distribution of $10000 \bar{p}(200 \mathrm{MeV} / c)$ obtained in ${ }^{4} \mathrm{He}$ gas at a temperature of $5.8 \mathrm{~K}$ and a pressure of $400 \mathrm{mbar}\left(\rho_{\mathrm{mol}}=8.66 \times 10^{-4}\right.$ $\left.\mathrm{mol} / \mathrm{cm}^{3}\right)$. The fraction of $\bar{p}$ stopping in the walls of the target chamber under these conditions is only about $1.1 \%$ and is not sensitive to possible uncertainties of the thicknesses of counters and windows in the beam line. The antiprotons were calculated to have a mean range of $8.6 \mathrm{~cm}$ and a range straggling of $3.6 \mathrm{~cm}$ [full width at half maximum (FWHM)] in the target cell. The situation changes drastically when the pressure is decreased to 200 mbar [Fig. 4(b)]. According to our simulation the mean range of antiprotons in the target gas at these conditions is $16.9 \mathrm{~cm}$, with a range
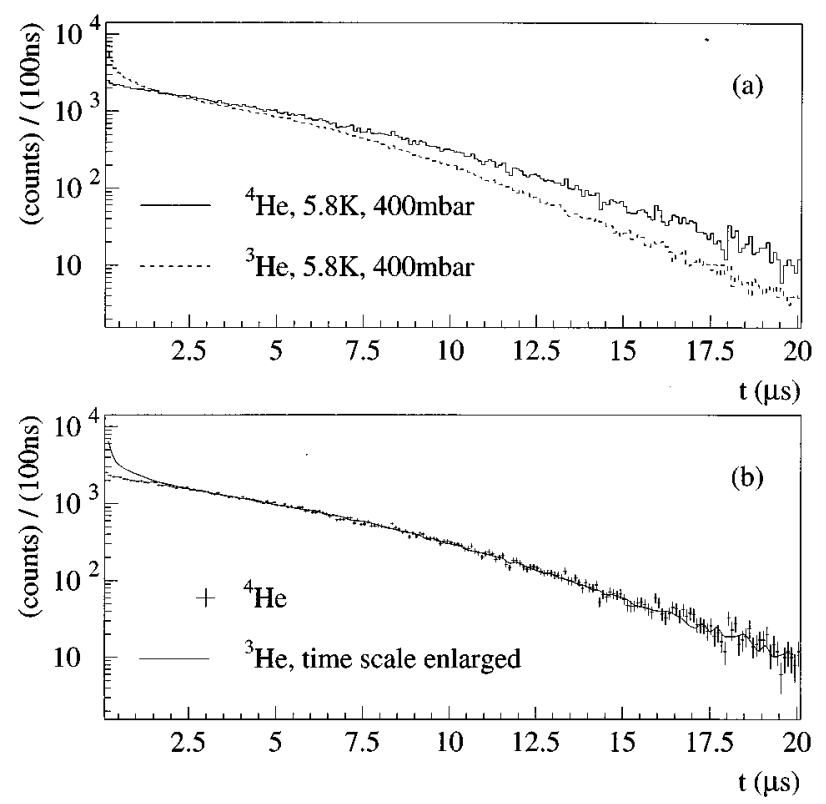

FIG. 5. Time spectra of the delayed $\bar{p}$ annihilation in ${ }^{3} \mathrm{He}$ (solid line) and ${ }^{4} \mathrm{He}$ (dashed line). (a) reveals the small difference in the time scale between ${ }^{3} \mathrm{He}$ and ${ }^{4} \mathrm{He}$ DATS for $t>2 \mu \mathrm{s}$. (b) shows the same spectra as (a), with the time scale of the ${ }^{3} \mathrm{He}$ spectrum enlarged by $14.6 \%$ (solid line) and the ${ }^{4} \mathrm{He}$ spectrum containing the errors of both spectra.

straggling of $9.5 \mathrm{~cm}$ (FWHM). About $50 \%$ of the antiprotons were then found to annihilate in the walls of the target chamber, thus considerably reducing the fraction of delayed $\bar{p}$. Unlike the situation at 400 mbar, however, this number depends strongly on the exact thicknesses of counters and windows in the beam line. Nevertheless, the simulation clearly shows that most antiprotons are stopped inside the target at $5.8 \mathrm{~K}$ and 400 mbar. We therefore use the data at our experimental "standard" conditions, namely, 5.8 K and 400 mbar.

\section{B. Overall trapping time}

Figure 5(a) compares DATS from pure ${ }^{3} \mathrm{He}$ and ${ }^{4} \mathrm{He}$ at $5.8 \mathrm{~K}$ and 400 mbar, showing that the shapes of the spectra after $2 \mu \mathrm{s}$ are surprisingly similar to each other except for the time scale. A quantitative value for this difference can be obtained by enlarging the time scale of the ${ }^{3} \mathrm{He}$ spectrum, until the long-lived parts of the two spectra match [4]. In order not to be sensitive to different short-lived components, only the time range between $2 \mu \mathrm{s}$ and $17.5 \mu$ s was taken into account. Figure 5(b) shows the same spectra as Fig. 5(a), with the time scale of the ${ }^{3} \mathrm{He}$ spectrum stretched by $14.6 \%$ (solid line) and the ${ }^{4} \mathrm{He}$ spectrum containing the errors of both spectra. From various pairs of DATS taken at the same conditions we obtained an average scaling factor of 1.144, which is readily interpreted as the ratio of overall trapping times

$$
\frac{T_{\text {trap }}\left({ }^{4} \mathrm{He}\right)}{T_{\text {trap }}\left({ }^{3} \mathrm{He}\right)}=1.144 \pm 0.009 .
$$

In previous publications $[4,8]$ the average lifetime $T_{\mathrm{av}}$ of antiprotons, calculated from the time spectra $N(t)$ as 


$$
T_{\mathrm{av}}\left(t_{0}, t_{\max }\right)=\frac{\int_{t_{0}}^{t_{\max }} t N(t) d t}{\int_{t_{0}}^{t_{\max }} N(t) d t}-t_{0},
$$

was used to compare DATS from ${ }^{3} \mathrm{He}$ and ${ }^{4} \mathrm{He}$. In order to consider only the long-lived part of the spectra, $t_{0}$ was chosen to be $2 \mu \mathrm{s}$ for ${ }^{3} \mathrm{He}$ and ${ }^{4} \mathrm{He}$. For a proper comparison of $T_{\mathrm{av}}\left({ }^{3} \mathrm{He}\right)$ and $T_{\mathrm{av}}\left({ }^{4} \mathrm{He}\right)$, however, one has to take into account that the time scale for ${ }^{4} \mathrm{He}$ was found to be a factor of 1.144 larger than for ${ }^{3} \mathrm{He}$. If $t_{0}$ is set to be $2 \mu \mathrm{s}$ for ${ }^{3} \mathrm{He}$, a value of $t_{0}=1.144 \times 2 \mu \mathrm{s} \simeq 2.3 \mu \mathrm{s}$ should therefore be used for ${ }^{4} \mathrm{He}$. Table I shows $T_{\mathrm{av}}\left({ }^{3} \mathrm{He}\right)$, calculated for $t_{0}=2 \mu \mathrm{s}$, while Table II contains $T_{\mathrm{av}}\left({ }^{4} \mathrm{He}\right)$ for $t_{0}=2.3 \mu \mathrm{s}$. At "standard" conditions the ratio of average lifetimes in ${ }^{4} \mathrm{He}$ and ${ }^{3} \mathrm{He}$ is

$$
\frac{T_{\mathrm{av}}\left({ }^{4} \mathrm{He}\right)}{T_{\mathrm{av}}\left({ }^{3} \mathrm{He}\right)}=1.153 \pm 0.009
$$

showing that $T_{\mathrm{av}}$ indeed reflects the overall trapping time of antiprotons, provided that the different time scales in ${ }^{3} \mathrm{He}$ and ${ }^{4} \mathrm{He}$ are taken into consideration.

\section{Short-lived component}

Apart from the small difference in the long-living part of the spectra a strong fast decaying component appears in all ${ }^{3} \mathrm{He}$ DATS, which is not present in low-density ${ }^{4} \mathrm{He}$ (Fig. 2 ). Although small admixtures of foreign gases (in particular $\mathrm{H}_{2}$ and $\mathrm{O}_{2}$ ) to helium are known to induce a fast component in the spectra [13], there is no possibility that gaseous impurities introduced into the present target could have caused the short-lived component in ${ }^{3} \mathrm{He}$ alone, because at temperatures around $5 \mathrm{~K}$ all possible impurities, e.g., $\mathrm{H}_{2}$ or $\mathrm{O}_{2}$, are frozen or liquefied. The presence of the fast-decaying component is therefore intrinsic to ${ }^{3} \mathrm{He}$. By fitting a simple sum of two exponential functions to the ${ }^{3} \mathrm{He}$ spectra between 0.1 $\mu \mathrm{s}$ and $5 \mu \mathrm{s}$, the lifetime of the fast component in ${ }^{3} \mathrm{He}$ was determined to be $0.154 \pm 0.007 \mu \mathrm{s}$. The fraction of all trapped antiprotons annihilating with this relatively short lifetime (compared to radiative transitions) was found to be $(13.3 \pm 0.2) \%$. This suggests that there is at least one state in $\bar{p}{ }^{3} \mathrm{He}^{+}$, which has an intermediate lifetime between metastability (lifetime $\sim 1-2 \mu \mathrm{s}$ ) and Auger dominance (lifetime $\lesssim 10 \mathrm{~ns})$.

\section{FITTING TO CASCADE MODELS}

In the preceding section the various overall trapping times of antiprotons in ${ }^{3} \mathrm{He}$ and ${ }^{4} \mathrm{He}$ have been determined by comparing DATS from ${ }^{3} \mathrm{He}$ and ${ }^{4} \mathrm{He}$ from $2 \mu \mathrm{s}$ up. These methods, with their sensitivity limited to the long-lived region, do not take into account the different short-lived components in ${ }^{3} \mathrm{He}$ and ${ }^{4} \mathrm{He}$. We therefore tried to find a fitting function which is capable of describing both the short- and long-lived components in the DATS. A propensity rule implies that, after being captured into a metastable state, the antiprotons follow a cascade through subsequent metastable levels with $\Delta n=\Delta l=-1[5,14]$. The DATS we observe are therefore a superposition of almost independent sequences, each with a radial node number $v=n-l-1=$ const. The

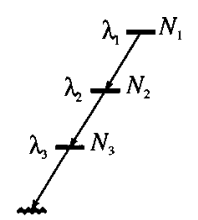

FIG. 6. Three-level cascade model. $\lambda_{1}, \lambda_{2}$, and $\lambda_{3}$ are the radiative decay rates of the metastable states. $N_{1}, N_{2}$, and $N_{3}$ represent the populations of the respective levels.

rates of crossover transitions between individual sequences have been estimated to be at least one order of magnitude smaller than the radiative transitions within a given chain [5]. As an approximation, one may describe the system of independent sequences by only one (fictitious) representative ladder of levels, averaging over the individual cascades.

\section{A. Three-level cascade model}

The simplest model which is able to reproduce the characteristic downward bending of the annihilation spectra at $t \sim 8 \mu \mathrm{s}$ is a $\bar{p}$ cascade over three metastable levels followed by an Auger-dominated short-lived state [8], as shown in Fig. 6. The antiproton annihilates immediately after the fast Auger transition to an ionized $\bar{p} \mathrm{He}^{2+}$ state. The rate equations for the populations of the different levels are then

$$
\begin{gathered}
\frac{d N_{1}}{d t}=-\lambda_{1} N_{1}, \\
\frac{d N_{2}}{d t}=-\lambda_{2} N_{2}+\lambda_{1} N_{1}, \\
\frac{d N_{3}}{d t}=-\lambda_{3} N_{3}+\lambda_{2} N_{2},
\end{gathered}
$$

with $\lambda_{i}$ being the radiative decay rates of the metastable states. Since our aim was only to reproduce the gross shape of the time spectra from $100 \mathrm{~ns}$ on, the Auger-dominated state at the end of the cascade was not taken into account in our models. Due to the nonorthogonality of real exponential functions it is difficult to obtain a consistent set of fit results with six free parameters. In most of the cases, however, we found similar values for the radiative rates $\lambda_{1}$ and $\lambda_{2}$ both in ${ }^{3} \mathrm{He}$ and ${ }^{4} \mathrm{He}$. Therefore we set $\lambda_{1}=\lambda_{2}$ and further assumed the initial populations of all three levels to be equal $\left[N_{1}(0)=N_{2}(0)=N_{3}(0) \equiv N_{0}\right]$. In view of the high level density and the small level spacing around $n=n_{0}$ this is probably justified. The solution for $N_{3}(t)$ of this system of differential equations with three free parameters is

$$
\begin{aligned}
N_{3}(t)= & N_{0}\left\{\frac{\lambda_{1}^{2} t}{\lambda_{3}-\lambda_{1}}-\frac{\lambda_{1}^{2}}{\left(\lambda_{3}-\lambda_{1}\right)^{2}}+\frac{\lambda_{1}}{\lambda_{3}-\lambda_{1}}\right\} e^{-\lambda_{1} t} \\
& +N_{0}\left\{1-\frac{\lambda_{1}}{\lambda_{3}-\lambda_{1}}+\frac{\lambda_{1}^{2}}{\left(\lambda_{3}-\lambda_{1}\right)^{2}}\right\} e^{-\lambda_{3} t} .
\end{aligned}
$$

Figure 7 shows the results of a fit of the annihilation rate $\lambda_{3} N_{3}(t)$ to typical spectra, recorded at $5.8 \mathrm{~K}$ and $400 \mathrm{mbar}$ in ${ }^{3} \mathrm{He}$ and ${ }^{4} \mathrm{He}$. The shape of DATS from ${ }^{4} \mathrm{He}$ without a 


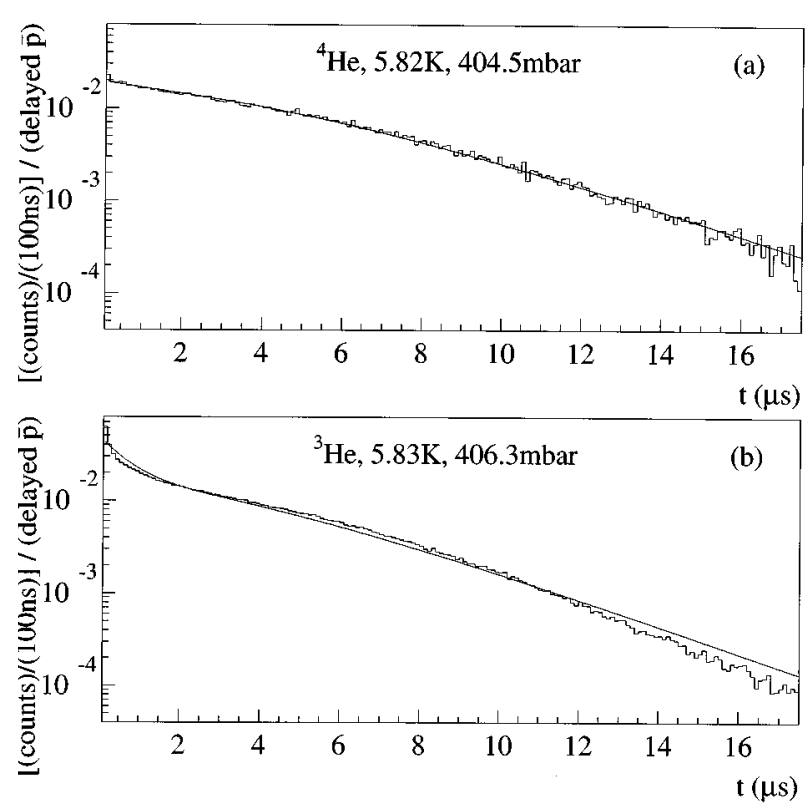

FIG. 7. Fits of a three-level model with three free parameters $\left(\lambda_{1}, \lambda_{3}\right.$, and $\left.N_{0}\right)$ to the spectra of Fig. 2.

fast component is described satisfactorily $\left(\chi_{\text {red }}^{2} \sim 2\right)$, while the simplified three-level fitting function cannot reproduce the strong short-lived component and the downward bent structure of $\ln N(t)$ for longer decay times in ${ }^{3} \mathrm{He}$ $\left(\chi_{\text {red }}^{2} \sim 10\right)$. Despite this lack of sensitivity the fit results for $\lambda_{1}$ and $\lambda_{3}$ are presented in Tables I and II for comparison with earlier publications. The six-parameter fit provides a

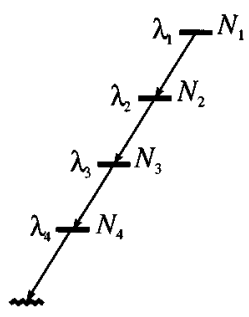

FIG. 8. Four-level model of the $\bar{p}$ cascade with decay rates $\lambda_{i}$ of the metastable states and level populations $N_{i}$, respectively.

better representation of ${ }^{3} \mathrm{He}$ DATS, but, as mentioned above, the fit results are inconsistent due to the large number of free parameters.

\section{B. Four-level cascade model}

One obvious possibility to enhance the growth-decay structure of the fitting function necessary for satisfactory fit results for ${ }^{3} \mathrm{He}$, is to consider a $\bar{p}$ cascade over four metastable states instead of three, as shown in Fig. 8. Since the downward bent shape of the DATS indicates a reduced feeding from higher levels at longer decay times, the initial population $N_{0}^{1}$ of the highest state in this four-level decay chain was introduced as an additional free parameter in the fit. The initial populations of the lower levels were assumed to be equal $\left(N_{0}\right)$. To ensure stable fit results, the decay rates were set to be equal for the three upper levels, as in the three-level model. Extending the three-level model as described results in a second growth-decay term $\propto t^{2} e^{-\lambda_{1} t}$ in addition to the one $\propto t e^{-\lambda_{1} t}$ in Eq. (10):

$$
\begin{aligned}
N_{4}(t)= & \left\{N_{0}^{1} \frac{\lambda_{1}^{3} t^{2}}{2\left(\lambda_{4}-\lambda_{1}\right)}-N_{0}^{1} \frac{\lambda_{1}^{3} t}{\left(\lambda_{4}-\lambda_{1}\right)^{2}}+N_{0} \frac{\lambda_{1}^{2} t}{\lambda_{4}-\lambda_{1}}\right\} e^{-\lambda_{1} t}+N_{0}\left\{\frac{\lambda_{1}^{3}}{\left(\lambda_{4}-\lambda_{1}\right)^{3}}-\frac{\lambda_{1}^{2}}{\left(\lambda_{4}-\lambda_{1}\right)^{2}}+\frac{\lambda_{1}}{\lambda_{4}-\lambda_{1}}\right\} e^{-\lambda_{1} t} \\
& +N_{0}\left\{1-\frac{\lambda_{1}}{\lambda_{4}-\lambda_{1}}+\frac{\lambda_{1}^{2}}{\left(\lambda_{4}-\lambda_{1}\right)^{2}}-\frac{\lambda_{1}^{3}}{\left(\lambda_{4}-\lambda_{1}\right)^{3}}\right\} e^{-\lambda_{4} t} .
\end{aligned}
$$

Typical fits are presented in Fig. 9. The function is capable of reproducing the behavior of DATS from ${ }^{4} \mathrm{He}$ (a) and ${ }^{3} \mathrm{He}$ (b) both for short and long decay times $\left(\chi_{\text {red }}^{2} \sim 2\right)$. To achieve this, however, an additional parameter had to be introduced.

The results for $\lambda_{1}$ and $\lambda_{4}$ are given in Tables I and II. In Fig. 10 the radiative rates $\lambda_{1}, \lambda_{4}$ and the ratio of initial populations $N_{0}^{1} / N_{0}$ are plotted versus the molar density. The decay rate $\lambda_{1}$ of the upper metastable states was found to be higher for ${ }^{3} \mathrm{He}$ than for ${ }^{4} \mathrm{He}$ [Fig. 10(a)]. Comparing values of $\lambda_{1}$ for ${ }^{3} \mathrm{He}$ and ${ }^{4} \mathrm{He}$ at "standard" conditions one finds an average ratio of

$$
\frac{\lambda_{1}\left({ }^{3} \mathrm{He}\right)}{\lambda_{1}\left({ }^{4} \mathrm{He}\right)}=1.14 \pm 0.03,
$$

which is in good agreement with the ratio we have obtained from the direct comparison of DATS [Eq. (4)]. Thus $\lambda_{1}$ determines the overall trapping time in our model. It is interesting to see that the value of $\lambda_{1}$ found for ${ }^{4} \mathrm{He}$ $\left[0.46(1) \mu \mathrm{s}^{-1}\right]$ in such a simplified model is in agreement with the decay rate of the $(n, l)=(40,36)$ state [0.49(2) $\left.\mu \mathrm{s}^{-1}\right]$, determined by resonant deexcitation of antiprotons with laser light [10].

The decay rate of the last metastable level $\lambda_{4}$ in our model represents the fast-decaying component of DATS [Fig. 10(b)]; it is roughly a factor of 3 higher in ${ }^{3} \mathrm{He}$ than in ${ }^{4} \mathrm{He}$, again suggesting the existence of at least one border state with a lifetime between a metastable and an Augerdominated zone in ${ }^{3} \mathrm{He}$. However, no calculations of Auger rates have been performed for ${ }^{3} \mathrm{He}$ up to now. Again, the result for $\lambda_{4}$ in ${ }^{4} \mathrm{He}\left[0.78(2) \mu \mathrm{s}^{-1}\right.$ ] agrees surprisingly well with the value found for the state $(n, l)=(39,35)$ in our laser resonance experiment $\left[0.72(2) \mu \mathrm{s}^{-1}\right]$. 


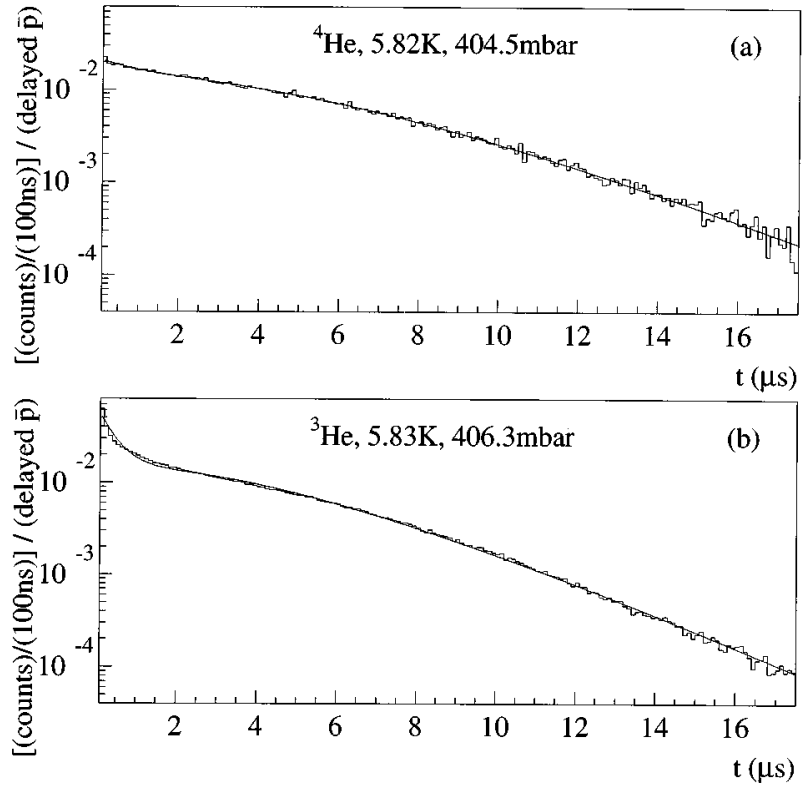

FIG. 9. Results of fitting a four-level cascade model with four free parameters to the spectra of Fig. 2.

The ratio of initial populations $N_{0}^{1} / N_{0}$, presented in Fig. 10 (c), is in all cases smaller than unity, indicating that the initial population of metastable states in a $\bar{p} \mathrm{He}^{+}$atom decreases with increasing principal quantum number $n$. Several arguments based on calculated transition rates show that an artificial cutoff in the initial population of states above $n \sim 40$ is necessary to reproduce the shape of DATS $[15,16]$.

\section{DISCUSSION AND CONCLUSIONS}

The observed isotope effects have to be compared with the present theoretical knowledge of $\bar{p} \mathrm{He}^{+}$atoms. The two main theoretical descriptions of this three-body system are the molecular approach $[15,17,18]$ and the atomic approach [5]. Very recently they have been supplemented by large configuration-space variational methods [19], which yielded the energy levels and transition wavelengths shown in Fig. 11. The rates for radiative transitions have been calculated both in the molecular and the atomic approaches. Up to now, the Auger rates have only been calculated for $\bar{p}{ }^{4} \mathrm{He}^{+}$, using the atomic model [5]. According to these calculations, Auger transitions with $\Delta l \leqslant 3$ to states of ionized $\bar{p} \mathrm{He}^{2+}$ (also shown in Fig. 11) occur quickly, the relevant states having lifetimes $\lesssim 10 \mathrm{~ns}$ (indicated by zigzag lines in Fig. 11). Auger transitions with $\Delta l>3$, on the other hand, are expected to be much slower even than the radiative processes and are thus "forbidden." The corresponding metastable levels have lifetimes on the order of 1-2 $\mu$ s (indicated by bold lines in Fig. 11). While actually calculated only for $\bar{p}{ }^{4} \mathrm{He}^{+}$, the same rule was applied to the case of $\bar{p}{ }^{3} \mathrm{He}^{+}$in Fig. 11(b).

The overall trapping time $T_{\text {trap }}$ of antiprotons depends on (i) the lifetimes of the individual metastable levels, (ii) the number of metastable states, and (iii) the initial population of these states. Assuming that the $\bar{p}$ is captured into a Bohr orbit with a principal quantum number $n \sim n_{0}=\sqrt{M^{*} / m_{\mathrm{e}}}$ (approximately 38 for ${ }^{4} \mathrm{He}$ and 37 for ${ }^{3} \mathrm{He}$ ) and with a life-

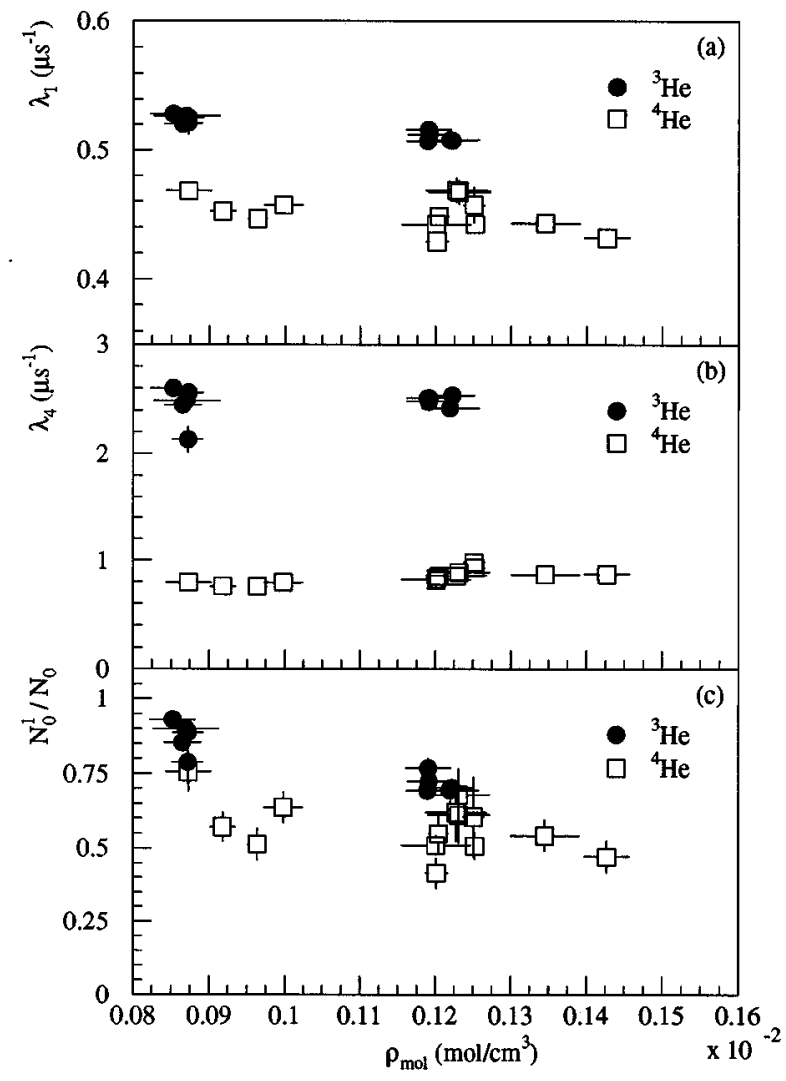

FIG. 10. Fit parameters $\lambda_{1}$ (a), $\lambda_{4}(\mathrm{~b})$, and $N / N_{0}$ (c) as calculated from the four-level cascade model for ${ }^{3} \mathrm{He}$ and ${ }^{4} \mathrm{He}$.

time $\tau_{n_{0}}$, the overall trapping time $T_{\text {trap }}$ can be estimated [5] by

$$
\begin{aligned}
& T_{\text {trap }} \propto \tau_{n_{0}} \times \text { number of metastable states } \\
& \propto\left[\frac{M^{*}}{m_{\mathrm{e}}}\right]^{3 / 2}\left[\frac{M^{*}}{m_{\mathrm{e}}}\right]^{1 / 2}=\left[\frac{M^{*}}{m_{\mathrm{e}}}\right]^{2} .
\end{aligned}
$$

This yields for the ratio of overall trapping times in ${ }^{4} \mathrm{He}$ and ${ }^{3} \mathrm{He}$

$$
\frac{T_{\text {trap }}\left({ }^{4} \mathrm{He}\right)}{T_{\text {trap }}\left({ }^{3} \mathrm{He}\right)} \approx\left[\frac{M^{*}\left(\bar{p}^{4} \mathrm{He}\right)}{M^{*}\left(\bar{p}^{3} \mathrm{He}\right)}\right]^{2} \simeq 1.14
$$

The experimental results for this ratio, as given in Eqs. (4) and (6), are in excellent agreement with this simple theoretical treatment based on the atomic model. A less direct approach to compare the DATS from ${ }^{4} \mathrm{He}$ and ${ }^{3} \mathrm{He}$ is to examine the fitted decay rates. The ratio we obtain from values of $\lambda_{1}$ found in the four-level model for ${ }^{3} \mathrm{He}$ and ${ }^{4} \mathrm{He}$ at similar densities [Eq. (12)] shows that $\lambda_{1}$ determines the overall trapping time in our model. We cannot extract decay rates of individual states from these simplified models, for which the ratio of lifetimes according to Eq. (13) would be 1.10. Therefore we have shown that the three different methods employed - scaling of the spectra, calculating the aver- 

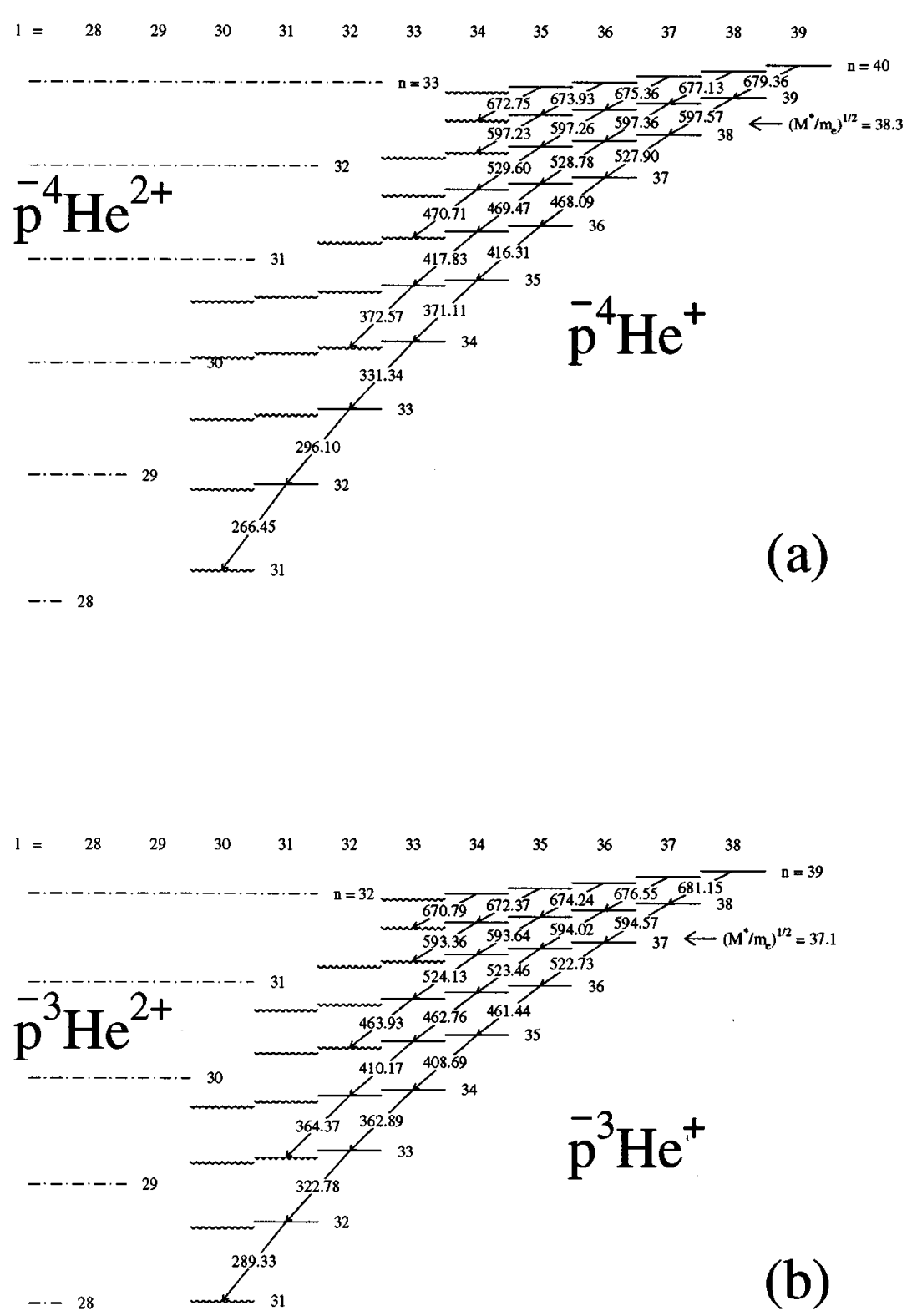

FIG. 11. Energy diagrams of $\bar{p}{ }^{4} \mathrm{He}^{+}$(a) and $\bar{p}{ }^{3} \mathrm{He}^{+}(\mathrm{b})$, together with those of $\bar{p}{ }^{4} \mathrm{He}^{2+}$ and $\bar{p}{ }^{3} \mathrm{He}^{2+}$. The states of $\bar{p} \mathrm{He}^{+}$are divided into a radiation-dominated metastable zone (bold lines) and a zone of fast-decaying states (zigzag lines), which are expected to decay to states of ionized $\bar{p} \mathrm{He}^{2+}$ via Auger transitions with $\Delta l \leqslant 3$. The main radiative cascades are indicated by arrows. The transition wavelengths calculated by Korobov [19] are shown for the relevant transitions, respectively. age lifetime, and fitting a chain decay model - converge to the same result for the overall trapping time as a simple theoretical estimate.

The fact that we have observed a pronounced short-lived component in ${ }^{3} \mathrm{He}$ DATS at $5.8 \mathrm{~K}$ and $400 \mathrm{mbar}$, which does not appear in ${ }^{4} \mathrm{He}$ under the same conditions, seems to indicate the existence of an initially populated state with an intermediate lifetime between the metastable zone and the Auger-dominated zone in ${ }^{3} \mathrm{He}$. Calculations of Augertransition rates for ${ }^{3} \mathrm{He}$, which might give an explanation for this striking effect, are therefore eagerly awaited.

We have observed a $22 \%$ lower trapping fraction of antiprotons in pure ${ }^{3} \mathrm{He}$ than in pure ${ }^{4} \mathrm{He}$ at low temperatures. A similar difference of the trapping fractions in roomtemperature gases was also reported in [4]. On the other hand, a crude theoretical estimate assuming a flat energy distribution of $\bar{p}$ up to $25 \mathrm{eV}$ before capture gives $f \approx 4 \%$ for helium, independent of the reduced mass of the $\bar{p} \mathrm{He}^{+}$system [5].

\section{ACKNOWLEDGMENTS}

We are indebted to the LEAR and PS staff at CERN for their tireless dedication in providing us with the high-quality antiproton beam. We would like to thank M. Arzberger for his help during the experiment. We are very grateful to $\mathrm{K}$. Ohtsuki, I. Shimamura, and V. I. Korobov for many valuable discussions and theoretical results. The present work is supported by the Grants-in-Aid for Specially Promoted Research and for International Scientific Research of the Japanese Ministry of Education, Science and Culture, the Japan Society for the Promotion of Science (JSPS), the Bundesministerium für Forschung und Technologie, and the Hungarian National Science Foundation. F.E.M. acknowledges financial support from INOUE. 
[1] M. Iwasaki, S.N. Nakamura, K. Shigaki, Y. Shimizu, H. Tamura, T. Ishikawa, R.S. Hayano, E. Takada, E. Widmann, H. Outa, M. Aoki, P. Kitching, and T. Yamazaki, Phys. Rev. Lett. 67, 1246 (1991).

[2] R.S. Hayano, M. Iwasaki, and T. Yamazaki, in Perspectives of Meson Science, edited by T. Yamazaki, K. Nakai, and K. Nagamine (North-Holland, Amsterdam, 1992), p. 417.

[3] T. Yamazaki, E. Widmann, R.S. Hayano, M. Iwasaki, S.N. Nakamura, K. Shigaki, F.J. Hartmann, H. Daniel, T. von Egidy, P. Hofmann, Y.-S. Kim, and J. Eades, Nature (London) 361, 238 (1993).

[4] S.N. Nakamura, R.S. Hayano, M. Iwasaki, K. Shigaki, E. Widmann, T. Yamazaki, H. Daniel, T. von Egidy, F.J. Hartmann, P. Hofmann, Y.-S. Kim, and J. Eades, Phys. Rev. A 49, 4457 (1994).

[5] T. Yamazaki and K. Ohtsuki, Phys. Rev. A 45, 7782 (1992).

[6] G.T. Condo, Phys. Lett. 9, 65 (1964).

[7] J.E. Russell, Phys. Rev. Lett. 23, 63 (1969); Phys. Rev. 188, 187 (1969); Phys. Rev. A 1, 721 (1970); 1, 735 (1970); 1, 742 (1970); J. Math. Phys. 12, 1906 (1971); Phys. Rev. A 6, 2488 (1972).

[8] E. Widmann, I. Sugai, T. Yamazaki, R.S. Hayano, M. Iwasaki, S.N. Nakamura, H. Tamura, T.M. Ito, A. Kawachi, N. Nishida, W. Higemoto, Y. Ito, N. Morita, F.J. Hartmann, H. Daniel, T. von Egidy, W. Schmid, J. Hoffmann, and J. Eades, Phys. Rev. A 51, 2870 (1995).

[9] N. Morita, M. Kamakura, T. Yamazaki, E. Widmann, H. Masuda, I. Sugai, R.S. Hayano, F.E. Maas, H.A. Torii, F.J. Hart- mann, H. Daniel, T. von Egidy, B. Ketzer, W. Müller, W. Schmid, D. Horváth, and J. Eades, Phys. Rev. Lett. 72, 1180 (1994).

[10] R.S. Hayano, F.E. Maas, H.A. Torii, N. Morita, M. Kamakura, T. Yamazaki, H. Masuda, I. Sugai, F.J. Hartmann, H. Daniel, T. von Egidy, B. Ketzer, W. Müller, W. Schmid, D. Horváth, J. Eades, and E. Widmann, Phys. Rev. Lett. 73, 1485 (1994); 73, 3181(E) (1994).

[11] W. Müller, F.J. Hartmann, J. Eades, R.S. Hayano, B. Ketzer, and F.E. Maas, Nucl. Instrum. Methods Phys. Res. Sect. A 349, 307 (1994).

[12] J.E. Kilpatrick, W.E. Keller, E.F. Hammel, and N. Metropolis, Phys. Rev. 94, 1103 (1954).

[13] E. Widmann, H. Daniel, J. Eades, T. von Egidy, F.J. Hartmann, R.S. Hayano, W. Higemoto, J. Hoffmann, T.M. Ito, Y. Ito, M. Iwasaki, A. Kawachi, N. Morita, S.N. Nakamura, N. Nishida, W. Schmid, I. Sugai, H. Tamura, and T. Yamazaki, Nucl. Phys. A558, 679c-688c (1993).

[14] I. Shimamura, Phys. Rev. A 46, 3776 (1992).

[15] V.E. Markushin, Yad. Fiz. 57, 1835 (1994).

[16] G. Ya. Korenman (unpublished) [see D. Horváth, Europhys. News 26, 57 (1995)].

[17] P.T. Greenland and R. Thürwächter, Hyperfine Interact. 76, 355 (1993).

[18] R. Ahlrichs, O. Dumbraijs, H. Pilkuhn, and H.G. Schlaile, Z. Phys. A 306, 297 (1982).

[19] V.I. Korobov (private communication). 